\title{
A PRINCIPLE GOVERNING THE DISTRIBUTION OF CURRENT IN SYSTEMS OF LINEAR CONDUCTORS
}

\author{
By F. Wenner
}

\section{ABSTRACT}

In a system of linear conductors in which the current in every branch is proportional to the impressed electromotive force, the current in any branch is that which would result should an electromotive force, equal to the potential difference which would appear across the break were the branch opened, be introduced into the branch and all other electromotive forces be removed.

The use of this principle leads to a material simplification in the solution of many of that class of problems for which it is generally considered that a solution may be obtained only by the application of Kirchhoff's laws. It may also be used to advantage in the solution of other moderately complicated problems involving the distribution of current.

\section{CONTENTS}

I. Introduction - -

II. Statement and proof _.

III. Applications and advantages . . . .

Problem 1._.

Problem 2 2

Problem 3...

Problem 4...

Problem 5...

Problem 6... 201

Problem 7 .

Problem 8.......... 204

IV. Summary

V. Appendix .......... 207

\section{INTRODUCTION}

In electrical measurements and the distribution of electric power it is often necessary to estimate or determine the current to be expected in one or more branches of a system of conductors in advance of assembly or construction, or when conditions are such that a direct measurement is not practicable. A case in point is the determination of the current in the galvanometer branch of a Wheatstone bridge network for a giren set of conditions.

In the discussion of this subject it will be presumed, at least for the most part, that the current is direct so tinat currents and electromotive forces may be added algebraically and the resistances only 
of the branches need be considered. Howerer, it should be understood that the same procedures may be followed when the currents are alternating, provided currents and electromotive forces are considered to be vectors and complex impedance operators are used instead of the resistances.

A straightforward and obvious procedure for the solution of the problem stated above and similar problems, originally proposed by Kirchhoff, 1 is to apply Ohm's law or Kirchhoff's second law to $n-m+1$ of the closed meshes or circuits of the system and Kirchhoff's first law ${ }^{2}$ to $m-1$ of the branch points, where $m$ is the number of branch points and $n$ the number of branches. This procedure gives $n$ simultaneous equations from which the current in each of the $n$ branches may be determined in terms of the electromotive force of the battery and the resistances. However, since for the simplest bridge $n$ is 6 , it will be seen that for many cases the algebra involved in the solution of the $n$ simultaneous equations will be long and tedious. Therefore, attempts have been made to develop less complicated procedures and these have led to a marked improvement in the situation.

By the use of mesh or cyclic currents, which is a special application of the principle of superposition, ${ }^{3}$ instead of Kirchhoff's first law, Maxwell ${ }^{4}$ has shown that a partial solution may be obtained from $n-m+1$ simultaneous equations and that a complete solution may be obtained simply by adding expressions given by the partial solution. A very thorough discussion of the subject based mainly on the contributions made by Kirchhoff and Maxwell is giren by Feussner. ${ }^{5}$

Further, it has been noted that in some cases a knowledge of the current in one branch in terms of the current in another branch and the resistances will serve as well as a knowledge of the current in terms of the electromotive force of the battery and the resistances. In such cases the number of unknowns is $n-1$, and consequently a partial solution may be obtained from $n-m$ simultaneous equations. ${ }^{\circ}$ Therefore, a partial solution for the Wheatstone bridge network may be obtained from two simultaneous equations, whereas the procedure proposed by Kirchhoff requires six. A discussion of the subject, including the contributions made by Firchhoff, Maxwell, and Callendar, and extended so as to apply when the current is alternating is given by Hague. ${ }^{7}$

\footnotetext{
1 Kirchhoff, Pogg. Ann.,72 , p. 497; 1847. Ges. Abh., p. 22.

2 Kirchhoff, Pogg. Ann., 64, p. 512; 1845. Ges. Abh., p. 15.

8 Helmholtz, Pogg. Ann., 89, p. 211; 1853. Ges. Abh., 1, p. 476. See also, Hausrath, Die.Untersuchung Elektrischer Systeme, p. 1; 1907.

- Maxwell, Electricity and Magnetism, 3d ed., 1, pp. 406, 475.

$\checkmark$ Feussner, Ann. d. Physik, 314, p. 1304; 1902.

- Callendar, Proc. Phys. Soc., 22, p. 222; 1909.

7 Hague, A. C. Bridge Measurements, chap. 2; 1923.
} 
In still other cases it is sufficient if the currents are known only approximately or when some special relation exists between the resistances. In many such cases it is possible to avoid entirely the use of simultaneous equations. For example, in a particular case it may be obvious that the current in one branch is small in comparison with the currents in other branches, and that if this branch were removed the system would be changed from a network to a group of series-parallel conductors. The current in all. branches except the one may then be calculated as though this one were not a part of the system, and the values thus obtained will not be materially in error. In some cases too it is possible to determine to a close approximation the current in that branch in which the current is small in comparison with the currents in other branches. Illustrations of this are the determination of the sensitivity of the Thomson bridge by Northrup, ${ }^{8}$ and the determination of the sensitivity of the Wheatstone and other bridges, potentiometers, etc., by Smith. ${ }^{9}$ In these determinations both considered the current through the galvanometer branch when the bridge is very nearly balanced to be that which would result from an electromotive force in one of the arms equal to the current in this arm times the change in resistance in this arm necessary to reduce the current in the galvanometer branch to zero. A proof of this principle, given by Smith, is based upon Kirchhoff's reciprocal theorem, ${ }^{10}$ a theorem which may be used to advantage in the solution of many problems in current distribution.

Another theorem of importance in this connection pertains to the equivalence of stars and deltas in systems of linear conductors. Kennelly ${ }^{11}$ has shown that any three-point star (three-way branchpoint) may be replaced by a delta or triangle and conversely any delta may be replaced by a three-point star. Further Rosen ${ }^{12}$ has shown the equivalence of any $n$-point star and a delta or network in which there are $1 / 2 n(n-1)$ conductors connecting each of the $n$ points to every other point. The use of this theorem leads to a material simplification in the calculation of the resistance or impedance be-tween any two points of a network. The theorem also has other applications.

In this brief discussion of the subject, reference has been made to most of the laws, principles, theorems, and procedures which pertain to this particular field of investigation. There is, however, another general principle governing the distribution of current in systems of

8 Northrup, Methods of Measuring Electrical Resistance, p. 121; 1912.

- Smith, B. A. Report, p. 3; 1906. Glazebrook, Dictionary of Applied Physies.

${ }^{10}$ Kirchhoff, Pogg. Ann., 72; 1847. Ges. Abh., p. 32. Maxwell, Electricity and Magnetism, 3d ed.,

1; p. 405. Jeans, Electricity and Magnetism, 3d ed., p. 327 .

11 Kennelly, Elec. World and Eng., 34, p. 413; 1899.

12 Rosen, Jour. I. E. E., 62, p. 916; 1924. 
linear conductors, which in many cases leads more directly to the solution of problems than does any of those referred to above.

This principle may have been applied to direct-current bridges ${ }^{13}$ by Jaeger, Lindeck, and Diesselhorst; it has been applied to alternating-current bridges ${ }^{14}$ and to other problems ${ }^{15}$ by myself, and with direct currents has been applied to a general network ${ }^{16}$ by Harrison and Foote. However, except as applied to simple circuits, it seems to be practically unknown. The purpose of this paper is to state this principle, to prove its validity, to show to what types of problems it is applicable, and to show the advantages to be gained by its use.

\section{STATEMENT AND PROOF}

Let it be assumed that the system of conductors to be considered is one in which:

(1) Each branch is linear. (2) Ohm's law is applicable to each branch. (3) Impressed electromotive forces may be distributed in any manner throughout the system.

For such a system of conductors, or that described in an appendix to this paper, the current in any branch is that which would result should an electromotive force, equal to the potential difference which would appear across the break were the branch opened, be introduced into the branch and all other electromotive forces be removed.

This principle may be developed from well-known laws and principles as follows:

1. From Ohm's law and the principle of superposition it follows that each electromotive force causes a current in each branch proportional to itself and independent of the currents caused by other electromotive forces, and the current in any branch is the algebraic sum of the currents in that branch caused by the various electromotive forces.

2. From 1 it follows that if in any branch there is introduced an .electromotive force of the proper sign and magnitude to reduce the current in this branch to zero, the current in this branch caused by this electromotive force is equal in magnitude but opposite in sign to the current in this branch caused by the other electromotive forces in the system.

3. With the current in any branch equal to zero the branch may be opened without changing the current in any branch or the potential difference between any two points in the system. Consequently, for the conditions given in 2 , opening the branch causes no potential difference to appear across the break.

13 Jaeger, Lindeck, and Diesselhorst, Zs. f. Inst., 23, p. 75; 1903.

${ }_{14}$ Wenner, Bull. Bur. Stds., 6, p. 369; 1909 (reprint No. 134).

16 Wenner, Jour. Opt. Soc. of Am. and Rev. Sci. Inst., 11, p. 495; 1925.

${ }^{16}$ Earrison and Foote, Trans. Am. Inst. Elec. Engr., 39, p. 390; 1920. 
4. With the hranch open any or all of the electromotive forces in it may be removed without changing the current in any branch of the system, but with the appearance of a potential difference across the break equal in magnitude but opposite in sign to the electromotive forces removerl.

5. Consequently, when a hranch is opened the potential difference which appears across the break is equal in magnitude but opposite in sign to the electromotive force which, if placed in this branch, would reduce the current to zero.

6. Therefore, if an electromotive force, equal to the potential difference which would appear across the break should a branch be opened, were introduced into the branch under consideration and all other electromotire forces regardless of their location be remored, learing all branches closed, the current in it would be the same as that which is caused by the other electromotive forces.

As a result of discussions with my colleagues, independent proofs of this principle have been dereloped by Dr. A. S. McAllister and Dr. Chester Snow. These unpublished proofs differ from each other, from that given by Harrison and Foote, and from that given above.

From the principle as stated and the principle of superposition it follows that:

1. For systems of conductors, such as those described above or in the appendix and in which there may be any number of electromotive forces, the change in current in any branch brought about by the introduction of an additional electromotive force is equal to the change in current which would be produced by the introduction into the particular branch of an electromotive force equal to the change which would occur in the potential difference across the break if such a break were present at the time the additional electromotive force is introduced.

2. For systems of conductors, such as are described abore or in the appendix the change in the current in any branch caused by the addition of a branch containing no electromotive force is equal to the change in current which would be produced in the particular branch by an electromotive force in the added branch equal to the potential difference which existed between the points to which the branch is connected, before the connection was made.

3. For systems of conductors, such as those described abore or in the appendix, except that in one branch the current is not proportional to the impressed electromotive force, the current in this branch is that which would result from an electromotive force, located in it, equal to the potential difference which would appear across the break were the branch opened. 


\section{APPLICATIONS AND ADVANTAGES}

To show something of the conditions under which the principle is applicable and the advantages to be gained by its use it will be applied in the solution of a number of problems. As these are to serve for illustration only, no special effort will be made to get the solutions into the most convenient form for use or to bring out any particular points pertaining to the problems.

\section{PROBLEM 1}

To measure the potential difference, $V$, between two terminals of a system of conductors by means of a voltmeter when conditions are such that the connection of the voltmeter causes an appreciable lowering of the potential difference. One of two procedures for making such a measurement, proposed by Brooks, ${ }^{17}$ is $(a)$ connect a voltmeter to the terminals and note the reading $V_{1} ;(b)$ insert in series with the voltmeter a resistance equal to the resistance of the voltmeter and note the reading $V_{2} ;(c)$ calculate $V$ from the equation

$$
V=V_{1} V_{2} /\left(V_{1}-V_{2}\right)
$$

To show that this procedure gives a correct result let:

$i_{1}$ be the current through the voltmeter when the reading is $V_{1}$, $i_{2}$ be the current through the voltmeter when the reading is $V_{2}$, $r$ be the resistance of the voltmeter, and $R$ be the resistance of the system of conductors, between the terminals between which the potential difference is to be determined.

According to the principle under consideration

$$
i_{1}=V /(\mathrm{R}+r) \text { and } i_{2}=V /(R+2 r)
$$

while the instrument is so graduated that

Therefore

$$
i_{1}=V_{1} / r \text { and } i_{2}=V_{2} / r
$$

$$
V=V_{1}(1+R / r)=V_{2}(2+R / r)
$$

from which it follows on the elimination of $R / r$ that

$$
V=V_{1} V_{2} /\left(V_{1}-V_{2}\right)
$$

\section{PROBLEM 2}

One of two 2-wire feeders supplying power from the same source to adjacent territories is found to be overloaded, and it is desired to know what shift in load might be expected were a 2-wire tie to be made between these. The conditions of the problem are as follows:

(a) Each feeder is transmitting power nominally at 13,000 rolts.

(b) At what seems to be a farorable place for making the tie the voltage of the excessively loaded feeder, found by measurement, is 260 rolts less than that of the other feeder. 
(c) The resistance (or impedance to an electromotive force located in the tie estimated from the size and length of the conductors proposed for the tio and the size and length of the feeders is $3.25 \mathrm{ohms}$.

Therefore, according to the principle under eonsideration, making the tie would cause a shift of $260 / 3.25$ or 80 amperes from one feeder to the other and would relieve the excessively loaded feeder to the extent of $80 \times 13,000$ volt-amperes or 1,040 k.v.a.

As a simple independent analytical method for solving this problem was not evident the principle was applied to other problems of the same type, and the results obtained checked experimentally.

\section{PROBLEM 3}

To determine the current through the galvanometer oranch of an approximately balınced Wheatstone bridge network.

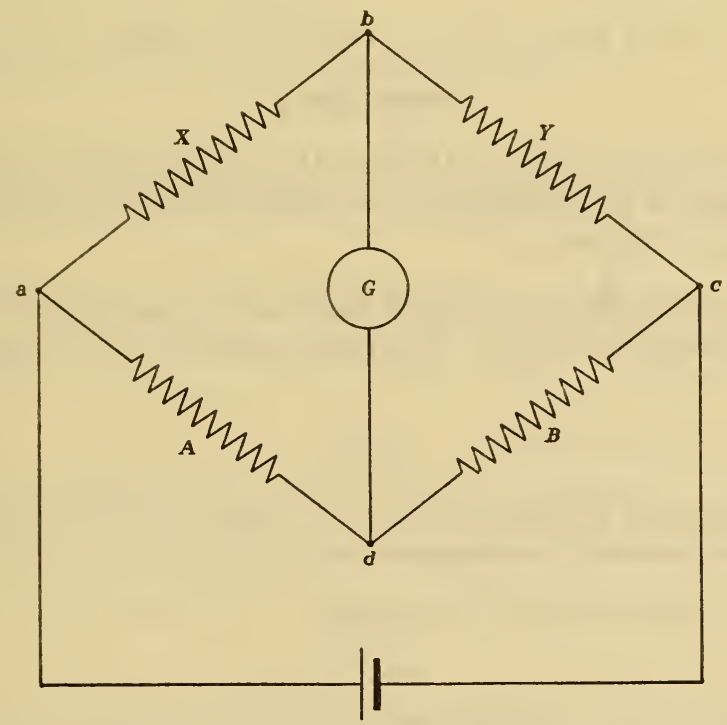

Fig. 1.-Wheatstone bridge

Referring to Figure 1, let

$E$ be the potential drop from $(a)$ to $(c)$ with the galvanometer branch open,

$X, Y, A$, and $B$ be the resistances of the arms of the bridge,

$G$ be the resistance of the galvanometer branch,

$R$ be the resistance of the galvanometer circuit; that is, the resistance to an electromotive force in the galvanometer branch, and

$i_{\mathrm{g}}$ be the current in the galvanometer branch.

$90587^{\circ}-26-2$ 
With the galvanometer branch open it may be seen by inspection that the potential drop from $a$ to $b$ is

$$
\begin{aligned}
& E X /(X+Y) \text { and from } a \text { to } d \text { is } \\
& E A /(A+B)
\end{aligned}
$$

The potential difference across the break in the galvanometer branch then is

$$
E X /(X+Y)-E A /(A+B) \text { or } E(X B-Y A) /(X+Y)(A+B)
$$

Therefore, if the galvanometer branch is closed

$$
i_{\mathrm{g}}=E(X B-Y A) /(X+Y)(A+B) R
$$

If this bridge is approximately balanced, which is the case usually to be considered,

$$
\begin{gathered}
R=G+X Y /(X+Y+)+A B /(A+B), \text { approximately. } \\
\text { PROBLEM } 4
\end{gathered}
$$

To determine the current through the galvanometer branch of the Brooks model 5 potentiometer, ${ }^{18}$ for which the circuit arrangement is that shown in Figure 2.

To solve this problem consider first that the galvanometer branch and the $r_{1}$ branch are open. Then the current in the $r_{6}$ branch will be

$$
\frac{e_{1}}{r_{3}+r_{8}}
$$

and the potential difference across the break in the $r_{1}$ branch will be this current times the resistance $r_{6}$ or

$$
\frac{e_{1} r_{6}}{r_{3}+r_{6}}
$$

Now consider the $r_{1}$ branch closed, then the current in it will be this potential difference considered as an electromotive force dirided by the resistance, or

$$
\frac{\frac{e_{1} r_{6}}{r_{3}+r_{6}}}{r_{1}+r_{2}+r_{3} r_{6} /\left(r_{3}+r_{6}\right)}
$$

The potential difference across the break in the galvanometer branch caused by the electromotive force $e_{1}$ then will be this current times $r_{1}$ and that caused by the electromotive force $E$, which is opposite of sign, is the current $E / R$ time the resistance $R / p$. The potential 
difference across the break in the galvanometer branch, therefore, will be

$$
\frac{\frac{e_{1} r_{6} r_{1}}{r_{3}+r_{6}}}{r_{1}+r_{2}+r_{3} r_{6} /\left(r_{3}+r_{6}\right)}-\frac{E R}{R p}
$$

With the galvanometer branch closed, the current in the galvanometer branch will be this potential difference considered as an elec-

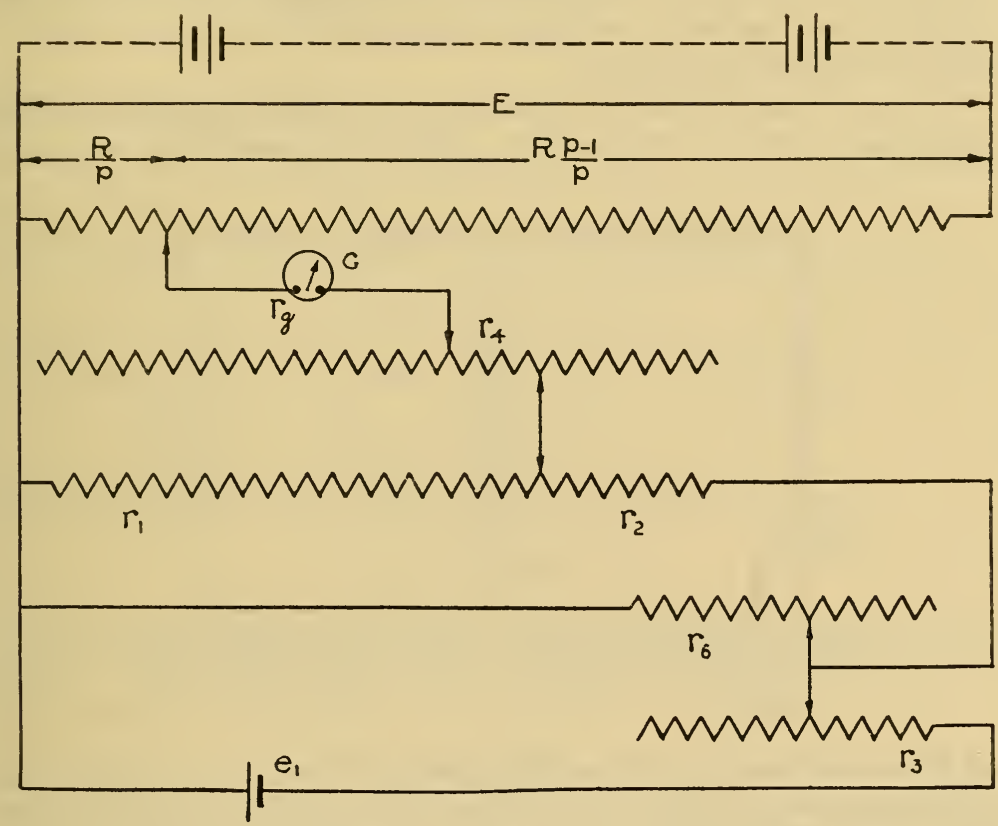

FIG. 2.-Circuits of Brooks model 5 deflection potentiometer

tromotive force divided by the resistance of the galvanometer circuit, or,

$$
i_{\mathrm{g}}=\frac{\frac{e_{1} r_{6} r_{1}}{r_{3}+r_{6}}}{r_{\mathrm{g}}+r_{4}+\frac{r_{1}\left[r_{2}+r_{3} r_{6} /\left(r_{3}+r_{6}\right)\right]}{r_{1}+r_{2}+r_{3} r_{6} /\left(r_{3}+r_{6}\right)}+\frac{E R}{R / p \times R(p-1) / p}}
$$

In the development of this equation terms once written down have not been changed even in those cases in which simplifications might readily have been made. Consequently it might have been written simply from an inspection of the diagram of connections and, in slighty different form, was so written on first consideration of the problem. The equation may readily be reduced to the simpler form given by Brooks. 


\section{PROBLEM 5}

To determine the values for the resistances of a shunt box for a galvanometer to satisfy the following conditions: (a) the current drawn from the source of the electromotive force after the deflection has reached a constant value shall be the same as though no shunt were used; $(b)$ the resistance to the electromotive force developed by the motion of the coil of the galvanometer shall be the same as though no shunt were used; $(c)$ the current through the galvanometer after the deflection has reached a constant value shall be $1 / n$ times what it would if no shunt were used.

Volkmann ${ }^{19}$ has shown that these conditions may be fulfilled by the arrangement shown in Figure 3 in which $X$ represents the resistance

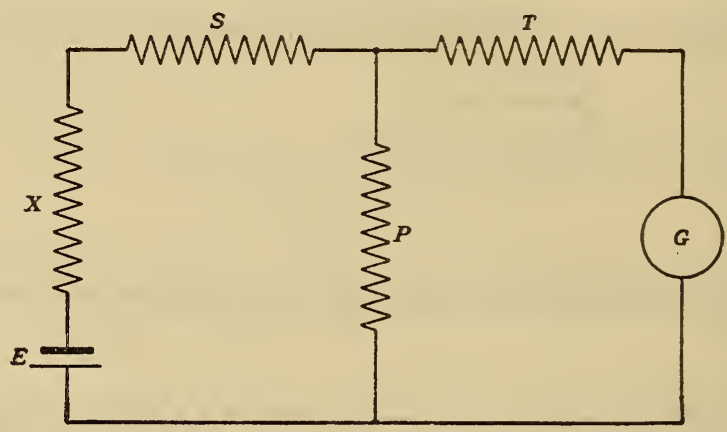

FIG. 3.-Shunt for reducing sensitivity of galvanometer while keeping the damping and current drawn from source constant

and $E$ the electromotive force of the source of the current, $G$ the resistance of the galvanometer, and $S, P$, and $T$ the resistances of the added conductors. Then if $I$ is the current drawn from the source and $i_{\mathrm{g}}$ is the current through the galvanometer, it follows from condition $(a)$ that

Therefore

$$
I=\frac{E}{X+S+P(T+G) /(P+T+G)}=\frac{E}{X+G}
$$

$$
S+P(T+G) /(P+T+G)=G
$$

From condition $(c)$ it follows that

$$
i_{\mathbf{g}}=\frac{\mathrm{E}}{X+S+P(T+G) /(P+T+G)} \times \frac{P}{P+T+G}=\frac{E}{n(X+G)}
$$

and dividing equation (3) by equation (1) gives

$$
P /(P+T+G)=1 / n
$$

10 Volkmann, Ann. der Physik, 12, p. 217, 1903. 
From the principle under consideration and condition (b) it follows that

$$
i_{\mathrm{g}}=E P /(P+S+X)(X+G)
$$

and substituting the expression for $i_{\mathrm{g}}$ given by equation (3) gives

$$
P /(P+X+S)=1 / n
$$

From equations (4) and (5) it follows that

$$
T=S+X-\mathrm{G}
$$

and from equation (5) it follows that

$$
P=(S+X) /(n-1)
$$

These expressions for $T$ and $P$ substituted in equation (2) give

$$
S=(G n-X) /(n+1)
$$

This expression for $S$ substituted in equation (5) gives

$$
P=n(X+G) /\left(n^{2}-1\right)
$$

and this expression for $P$ substituted in equation (4) gives

$$
T=(n X-G) /(n+1)
$$

These expressions for $S, P$, and $T$ are the same as those given by Edler ${ }^{20}$ who showed that the expressions given by Volkmann are unnecessarily complicated.

\section{PROBLEM 6}

To design a multiplier for a deflection instrument so that it may be used as a voltmeter with a range of 1.5 volts, when the only information arailable concerning its constants is that when $20 \mathrm{ohms}$ is placed in series the reading is proportional to the applied potential difference and a full-scale deflection is obtained for an applied potential difference of 0.15 volt. The problem, therefore, is to devise a system of conductors with the instrument connected into one branch, such that the current in this branch for any potential difference applied between a particular pair of terminals will be the same as for one-tenth this potential difference applied to the instrument with 20 ohms in series. A possible arrangement is that shown in Figure 4 in which values for the resistances $A$ and $B$ are so chosen that with the instrument branch open the potential difference across the break is one-tenth the potential drop from $m$ and $n$ and the resistance, as measured from the position of the instrument with the instrument

20 Edler, Elektrot, und Masch., 33, p. 165; 1915. 
removed and the terminals $m$ and $n$ connected by a conductor of negligible resistance, is $20 \mathrm{ohms}$. This gives

or

$$
B /(A+B)=0.1 \text { and } A B /(A+B)=20
$$

$$
A=200.0 \text { and } B=22.22
$$

Here the potential difference between $m$ and $n$ has been considered as though it were produced by a source of electromotive force having no resistance. However, as it is the potential difference and not the electromotive force which produces it that is to be measurad, the resistance or impedance of the source of the electromotive force does not enter into the problem.

In this case if the potential difference is alternating the impedance of the instrument may be presumed to increase with the deflection; consequently the current will not be proportional to the electromotive force. However, no limit need be stated or implied as to how nearly the current must be proportional to the applied electromotive force provided only that any increase in the electromotive force causes an increase in the current.

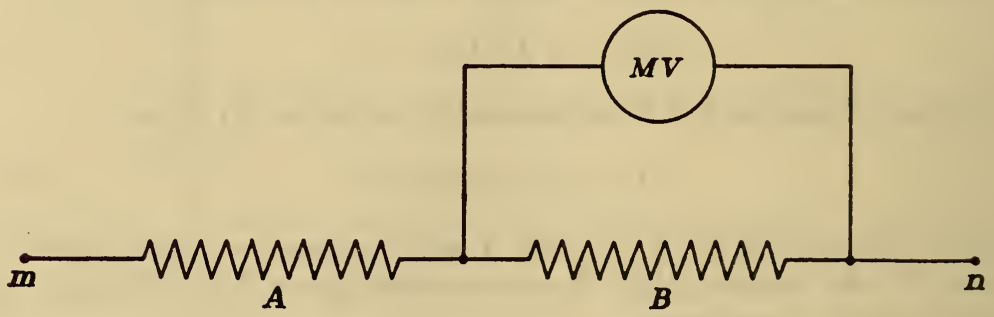

FIG. 4.-Multiplier for millivoltmeter

To show the application of the principle under consideration to somewhat more complicated problems it will be assumed that the currents are alternating. Here the symbolic notation will be used; that is, currents, potential differences, and electromotive forces will be represented by vectors, impedances will be represented by the complex operator $\boldsymbol{Z}$, and inductive coupling will be represented by the imaginary operator $\boldsymbol{M}$. Vectors, imaginary and complex quantities will be indicated by boldfaced type. For the more usual cases and

$$
\boldsymbol{M}=j \omega M
$$

where

$$
Z=R+j(\omega L-1 / \omega C)
$$

$j=\sqrt{-1}$

$\omega=2 \pi$ times the frequency

$M=$ mutual inductance

$R=$ resistance

$L=$ self-inductance

and

$C=$ capacitance. 
However, if a branch contains an instrument which develops a back electromotive force, $\boldsymbol{Z}$ must be understood to include the "motional" resistance and reactance as well as the electrical resistance and reactance.

\section{PROBLEM 7}

To determine the current through the detector branch of the Hughes balance, ${ }^{21}$ for which the eircuit arrangement is that shown

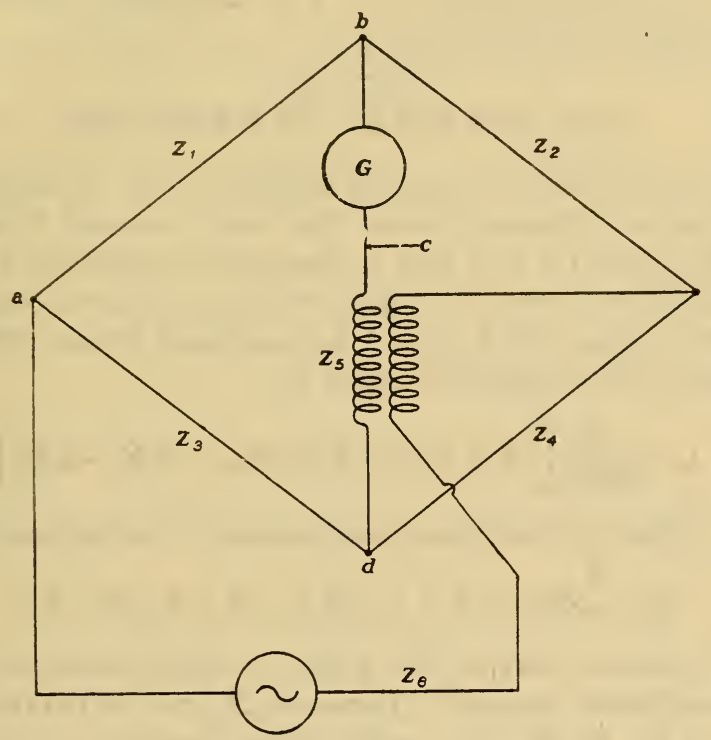

FIG. 5.-Hughes balance

in Figure 5. It is to be understood that the detector branch 5 and the generator branch 6 are connected by a mutual inductance and that each arm may be inductive.

Let

$\boldsymbol{E}$ be the electromotive force dereloped by the generator $\boldsymbol{i}_{\mathrm{g}}$ be the current in the detector branch,

$\boldsymbol{Z}_{1}, \boldsymbol{Z}_{2}, \boldsymbol{Z}_{3}, \boldsymbol{Z}_{4}, \boldsymbol{Z}_{5}$, and $\boldsymbol{Z}_{6}$ be the complex impedance operators for the branches, and $\boldsymbol{M}_{5-6}$ be the imaginary mutual operator for the branches 5 and 6 . With the detector branch open between $b$ and $c$ the total current is

$$
\frac{\boldsymbol{E}}{\boldsymbol{Z}_{6}+\left(\boldsymbol{Z}_{1}+\boldsymbol{Z}_{2}\right)\left(\boldsymbol{Z}_{3}+\boldsymbol{Z}_{4}\right) /\left(\boldsymbol{Z}_{1}+\boldsymbol{Z}_{2}+\boldsymbol{Z}_{3}+\boldsymbol{Z}_{4}\right)}
$$


and the drop in potential from $d$ to $c$ is this current times $\boldsymbol{M}$ or

$$
\frac{\boldsymbol{E}\left(\boldsymbol{Z}_{1}+\boldsymbol{Z}_{2}+\boldsymbol{Z}_{3}+\boldsymbol{Z}_{4}\right) \boldsymbol{M}_{5-6}}{\boldsymbol{Z}_{6}\left(\boldsymbol{Z}_{1}+\boldsymbol{Z}_{2}+\boldsymbol{Z}_{3}+\boldsymbol{Z}_{4}\right)+\left(\boldsymbol{Z}_{1}+\boldsymbol{Z}_{2}\right)\left(\boldsymbol{Z}_{3}+\boldsymbol{Z}_{4}\right)} \cdot
$$

The current through branches 3 and 4 is

$$
\frac{\boldsymbol{E}\left(\boldsymbol{Z}_{1}+\boldsymbol{Z}_{2}\right)}{\boldsymbol{Z}_{6}\left(\boldsymbol{Z}_{1}+\boldsymbol{Z}_{2}+\boldsymbol{Z}_{3}+\boldsymbol{Z}_{4}\right)+\left(\boldsymbol{Z}_{1}+\boldsymbol{Z}_{2}\right)\left(\boldsymbol{Z}_{3}+\boldsymbol{Z}_{4}\right)}
$$

and the potential drop from $a$ to $d$ is this current times $\boldsymbol{Z}_{3}$. The current through branches 1 and 2 is

$$
\frac{\boldsymbol{E}\left(\boldsymbol{Z}_{3}+\boldsymbol{Z}_{4}\right)}{\boldsymbol{Z}_{6}\left(\boldsymbol{Z}_{1}+\boldsymbol{Z}_{2}+\boldsymbol{Z}_{3}+\boldsymbol{Z}_{4}\right)+\left(\boldsymbol{Z}_{1}+\boldsymbol{Z}_{2}\right)\left(\boldsymbol{Z}_{3}+\boldsymbol{Z}_{4}\right)}
$$

and the potential drop from $a$ to $b$ is this current times $\boldsymbol{Z}_{1}$.

The potential difference across the break between $b$ and $c$ is the potential drop from $a$ to $d$ plus the potential drop from $d$ to $c$ minus the potential drop from $a$ to $b$ and, with the detector branch closed, the current is that which would be produced by an equal electromotive force in the detector branch, or

$$
\boldsymbol{i}_{\boldsymbol{\varepsilon}}=\frac{\boldsymbol{E}}{\boldsymbol{Z}_{\mathrm{m}} \boldsymbol{Z}^{2}{ }_{\mathrm{n}}}\left[\left(\boldsymbol{Z}_{1}+\boldsymbol{Z}_{2}+\boldsymbol{Z}_{3}+\boldsymbol{Z}_{4}\right) \boldsymbol{M}_{5-6}+\boldsymbol{Z}_{2} \boldsymbol{Z}_{3}-\boldsymbol{Z}_{1} \boldsymbol{Z}_{4}\right]
$$

where $\boldsymbol{Z}_{\mathrm{m}}$ is the complex impedance operator for the detector circuit and

$$
\boldsymbol{Z}^{2}{ }_{n}=\boldsymbol{Z}_{6}\left(\boldsymbol{Z}_{1}+\boldsymbol{Z}_{2}+\boldsymbol{Z}_{3}+\boldsymbol{Z}_{4}\right)+\left(\boldsymbol{Z}_{1}+\boldsymbol{Z}_{2}\right)\left(\boldsymbol{Z}_{3}+\boldsymbol{Z}_{4}\right)
$$

It should be noted that to this point no approximations have been made or conditions imposed. However, $\boldsymbol{Z}_{\mathrm{m}}$ can be expressed readily in terms of $\boldsymbol{Z}_{1}, \boldsymbol{Z}_{2}, \boldsymbol{Z}_{3}$, etc., only when the bridge is very nearly balanced or when the impedance of the generator circuit is very high in comparison with the impedance of the arms of the bridge. Then $\boldsymbol{Z}_{\mathrm{m}}$ may be considered to be the same as though the generator branch were open, in which case

$$
\boldsymbol{Z}_{\mathrm{m}}=\boldsymbol{Z}_{5}+\left(\boldsymbol{Z}_{1}+\boldsymbol{Z}_{3}\right)\left(\boldsymbol{Z}_{2}+\boldsymbol{Z}_{4}\right) /\left(\boldsymbol{Z}_{1}+\boldsymbol{Z}_{3}+\boldsymbol{Z}_{3}+\boldsymbol{Z}_{4}\right)
$$

Should a case arise in which the approximation made would not be justifiable it probably would be possible to measure $\boldsymbol{Z}_{\mathrm{m}}$ directly.

\section{PROBLEM 8}

To determine the current through the detector branch of the Anderson bridge ${ }^{22}$ when each branch is inductive. Referring to Figure 6, let

$\boldsymbol{E}$ be the potential drop from $a$ to $b$ with the detector branch open;

$\boldsymbol{i}_{\boldsymbol{g}}$ be the current through the detector branch, 
and

$\boldsymbol{Z}_{1}, \boldsymbol{Z}_{2}, \boldsymbol{Z}_{3}, \boldsymbol{Z}_{4}, \boldsymbol{Z}_{5}, \boldsymbol{Z}_{6}$, and $\boldsymbol{Z}_{7}$ be the complex impedance operators for the various branches. With the detector branch open it will be seen by inspection that the potential drop from $a$ to $c$ is

The current through $\boldsymbol{Z}_{4}$ is

$$
\frac{E Z_{1}}{Z_{1}+Z_{2}}
$$

$$
\frac{\boldsymbol{E}}{\boldsymbol{Z}_{4}+\boldsymbol{Z}_{3}\left(\boldsymbol{Z}_{6}+\boldsymbol{Z}_{7}\right) /\left(\boldsymbol{Z}_{3}+\boldsymbol{Z}_{6}+\boldsymbol{Z}_{7}\right)}=\frac{\boldsymbol{E}\left(\boldsymbol{Z}_{3}+\boldsymbol{Z}_{6}+\boldsymbol{Z}_{7}\right)}{\boldsymbol{Z}_{4}\left(\boldsymbol{Z}_{3}+\boldsymbol{Z}_{6}+\boldsymbol{Z}_{7}\right)+\boldsymbol{Z}_{3}\left(\boldsymbol{Z}_{6}+\boldsymbol{Z}_{7}\right)}
$$

and the fraction of this current passing through $Z_{7}$ is

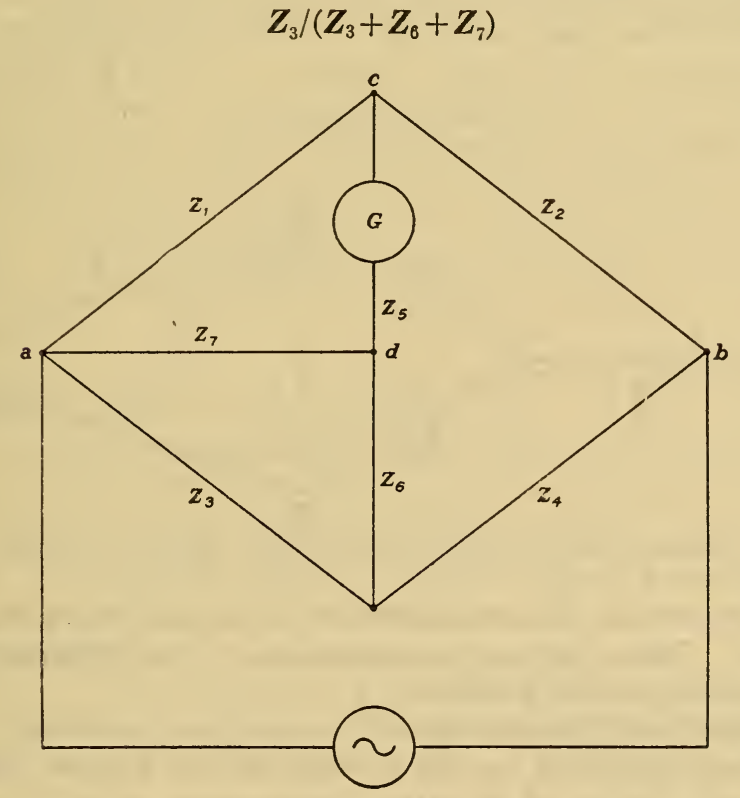

Fig. 6.-Anderson bridge

Therefore the current through $\boldsymbol{Z}_{7}$ times $\boldsymbol{Z}_{7}$, which is the drop in potential from $a$ to $d$, is

$$
\frac{\boldsymbol{E} \boldsymbol{Z}_{7} \boldsymbol{Z}_{3}}{\boldsymbol{Z}_{3}\left(\boldsymbol{Z}_{6}+\boldsymbol{Z}_{7}\right)+\boldsymbol{Z}_{\mathbf{4}}\left(\boldsymbol{Z}_{3}+\boldsymbol{Z}_{6}+\boldsymbol{Z}_{7}\right)}
$$

With the detector branch closed the current in it is the difference between these two open-circuit potential drops considered as an electromotive force in the detector branch divided by the impedance, $\boldsymbol{Z}_{\mathrm{m}}$, of the detector circuit, or

$$
i_{\mathrm{g}}=\frac{E}{Z_{\mathrm{m}}}\left[\frac{\boldsymbol{Z}_{1}}{Z_{1}+Z_{2}}-\frac{Z_{7} Z_{3}}{Z_{3}\left(Z_{7}+Z_{6}\right)+Z_{4}\left(Z_{3}+Z_{6}+Z_{7}\right)}\right]
$$


If the bridge is very nearly balanced, which is the only case of practical importance, or if the impedance of the generator branch is rery small in comparison with the impedance of the arms of the bridge, in writing an expression for $Z_{\mathrm{m}}$ the branch points, $a$ and $b$, may be considered to be connected by a conductor of negligible impedance. Referring then to Figure 7 it will be seen by inspection that

$$
\boldsymbol{Z}_{\mathrm{m}}=\boldsymbol{Z}_{5}+\frac{\boldsymbol{Z}_{1} \boldsymbol{Z}_{2}}{\boldsymbol{Z}_{1}+\boldsymbol{Z}_{2}}+\frac{\boldsymbol{Z}_{7}\left[\boldsymbol{Z}_{3} \boldsymbol{Z}_{4} /\left(\boldsymbol{Z}_{3}+\boldsymbol{Z}_{4}\right)+\boldsymbol{Z}_{6}\right]}{\boldsymbol{Z}_{7}+\boldsymbol{Z}_{3} \boldsymbol{Z}_{4} /\left(\boldsymbol{Z}_{3}+\boldsymbol{Z}_{4}\right)+\boldsymbol{Z}_{6}}
$$

The suggestion made with reference to the deternination of the impedance of the detector circuit in connection with problem 7 also applies here. Further, it would be possible in any case, by the use of Kennelly's delta to star transformation, to express the impedance in terms of the impedances of the eight branches. In case $\boldsymbol{E}$ represents the electromotive force developed by the generator instead of the potential drop between the current terminals $a$ and $b$ of the bridge, it may

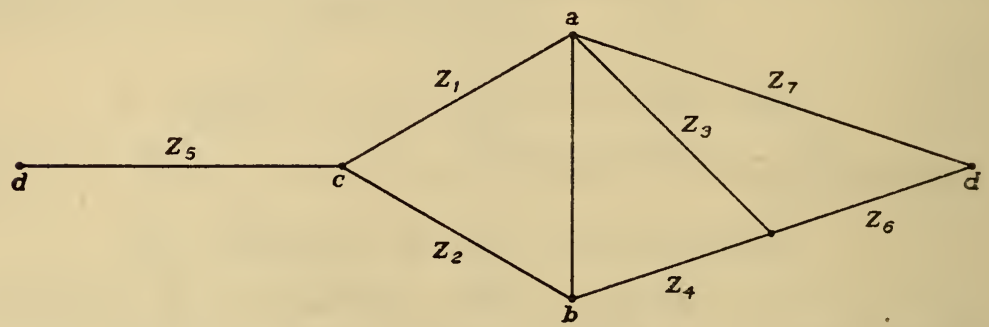

FIG. 7.-Anderson Bridge developed for showing impedance of detector circuit

be necessary to take into consideration the impedance of the generator branch. This adds some complications to the equations without serving any very useful purpose.

The application of this principle to a problem involving transient currents may be found in the Journal of the Optical Society of America and Review of Scientific Instruments to which reference has been made above. The problems considered should furnish a fairly definite suggestion as to the possible applications of the principle while a comparison of the solutions giren here with the solutions for the same problems given in text books or derived by any one of the more usual procedures should show the advantage to be gained by its use.

Before bringing this paper to a close I wish to state a personal point of view regarding it. As I consider the matter there is only one law, principle, or theorem giving the relation between the electromotive forces and the currents in a system of conductors, if of the usual type, which should be considered as fundamental. This was first definitely stated by $\mathrm{Ohm}$, approximately 100 years ago, and from time to time 
others have called attention to the types of conductors to which it is not applicable and hare shown how it must be interpreted in special cases, such, for example, as when the electromotive forces are alternating. This paper was written not to set forth a new or little understood principle, but to describe a procedure which I have been using in the application of Ohm's law to a limited number of problems, and to show that this procedure possesses certain advantages in comparison with other procedures, followed in the application of the same law to the same problems. The form of presentation was adopted in the hope of bringing this procedure to the attention of undergraduate students of physics, engineers, and those of us who either never have had or have forgotten that expert knowledge of determinants necessary for the expeditious handling of such problems by the "classical" procedure.

\section{SUMMARY}

1. A brief résumé is given of the procedures which have been proposed for determining the distribution of current in systems of linear conductors. In this connection reference is made to practically all of the laws, principles, and theorems which pertain to this field of investigation.

2. A new or at least not generally known principle is discussed. This principle may be stated as follows: In a system of linear conductors in which the current in every branch is proportional to the impressed electromotive force, the current in any branch is that which would result should an electromotive force, equal to the potential difference which would appear across the break were the branch opened, be introduced into the branch and all other electromotive forces be removed.

3. A proof of this principle is given and it is shown that, in some cases at least, its use leads more directly to a solution for the current in a branch of a network than does the use of Kirchhoff's laws.

\section{APPENDIX}

\section{SPECIFICATION FOR THE SYSTEM OF CONDUCTORS}

The following is a more complete specification for the system of conductors than that given on page 194 .

1. Each branch may contain a resistance, an inductance, or a capacitance or any two or more of these in series, a capacitance between parts of itself, may be inductively related to one or more of the other branches of the system, may be of sufficient cross section so the distribution of the current depends upon the time function of the impressed electromotive force, and may be so arranged as to move with respect to a constant magnetic field, thus developing a counter electromotive force. An example of the latter is a branch containing a galvanometer, a telephone receiver, or similar apparatus. 
2. The impressed electromotive forces may be arbitrary functions of time, and may have an arbitrary distribution throughout the system. For example, in one branch there may be a direct and constant electromotive force, in a second there may be an alternating electromotive force, in a third there may be an alternating electromotive force having an arbitrary phase relation to that in the second or a different frequency, in a fourth there may be a transient electromotive force, etc., or any two or more of the electromotive forces may be the same function of time or may be located in the same branch.

3. The current in each branch and the potential difference between each pair of points depends linearly upon all the applied electromotive forces. By this it is to be understood that should the introduction of an electromotive force, $E F(t)$, into a particular branch cause changes in the currents, amounting to $I_{1} F_{1}(t), I_{2} F_{2}(t), I_{3} F_{3}(t)$, etc., in the various branches, and cause changes in the potential differences amount to $V_{1} f_{1}(t), V_{2} f_{2}(t), V_{3} f_{3}(t)$, etc., between various pairs of points; the introduction of an electromotive force, $n E F(t)$, into the particular branch would cause changes in the currents amounting to $n I_{1} F_{1}(t), n I_{2} F_{2}(t)$, $n I_{3} F_{3}(t)$, etc., in the same branches, and cause changes in the potential differences amounting to $n V_{1} f_{1}(t), n V_{2} f_{2}(t), n V_{3} f_{3}(t)$, etc., between the same pairs of points. However, one branch, if it is the only one in which the current is to be determined, may be of such a type as to cause a departure from proportionality between currents, potential differences, and the applied electromotive forces.

For such a system of conductors, the principle and the proof for it applies as readily as they do when each branch contains resistance only, and all currents and electromotive forces are direct and constant.

Washington, February 25, 1926. 